BULL. AUSTRAL. MATH. SOC.

VOL. $10(1974), 169-175$.

\title{
Maximal Fitting classes of finite soluble groups
}

\section{R.A. Bryce and John Cossey}

From recent results of lausch, it is easy to establish necessary and sufficient conditions for a Fitting class to be maximal in the class of all finite soluble groups. We use Lausch's methods to show that there are normal Fitting classes not contained in any Fitting class maximal in the class of all finite soluble groups. We also find conditions on Fitting classes $\underline{\underline{X}}$ and $\underline{\underline{Y}}$ for $\underline{\underline{X}}$ to be maximal in $\underline{\underline{Y}}$.

1 .

Recall that a Fitting class is a class $\underline{\underline{X}}$ of (finite soluble) groups satisfying:

(i) if $G \in \underline{\underline{X}}$, and $N$ is a normal subgroup of $G$, then $N \in \underline{\underline{X}}$, and

(ii) if $G=N_{1} N_{2}$, where $N_{1}$ and $N_{2}$ are both normal in $G$, then $G \in \underline{\underline{\mathrm{X}}}$ if $N_{1}$ and $N_{2}$ are both in $\underline{\underline{\mathrm{x}}}$.

It follows from (ii) that for each Fitting class $\underline{\underline{x}}$ and group $G$, there is a largest normal subgroup $G_{\underline{X}}$ of $G$ which is in $\underline{\underline{X}}: G_{\underline{X}}$ is called

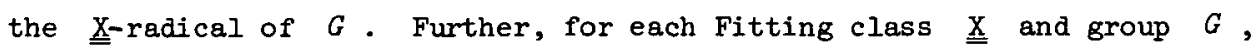
there is a subgroup $X$ of $G$ with the property that $X \cap N$ is a maximal X-subgroup of $N$, for every subnormal subgroup $N$ of $G$ : all subgroups with this property are conjugate, and they are called the $\underline{\underline{x} \text {-injectors of }}$ $G$ [4].

Received 16 October 1973. 
Given Fitting classes $\underline{\underline{X}}, \underline{\underline{Y}}$ with $\underline{\underline{X}} \subseteq \underline{\underline{Y}}$, we say that $\underline{\underline{X}}$ is normal in $\underline{\underline{Y}}$ if for every group $G \in \underline{\underline{Y}}$, the $\underline{\underline{X}}$-injectors of $G$ are normal, or, equivalently, $G_{\underline{\underline{X}}}$ is a maximal $\underline{\underline{X}}$-subgroup of $G$. If $\underline{\underline{X}}$ is normal in $\underline{\underline{S}}$, the class of all finite soluble groups, and $\underline{\underline{X}} \neq\{1\}$, we say $\underline{\underline{X}}$ is a normal Fitting class (note the departure here from the usage of [1]).

We will be concerned here with the following two problems: given Fitting classes $\underline{\underline{X}}, \underline{\underline{Y}}$ with $\underline{\underline{X}} \subset \underline{\underline{Y}}$, can we decide if $\underline{\underline{X}}$ is maximal in $\underline{\underline{Y}}$ (in the partial order given by inclusion), and can we always find a Fitting class $\underline{\underline{Z}}$ with $\underline{\underline{X}} \subseteq \underline{\underline{Z}} \subset \underline{\underline{Y}}$ and $\underline{\underline{Z}}$ maximal in $\underline{\underline{Y}}$ ?

If $\underline{\underline{X}}$ is maximal in $\underline{\underline{S}}$, we say $\underline{\underline{X}}$ is a maximal Fitting class. It may be deduced easily from the results of Lausch [6] that a normal Fitting class $\underline{\underline{x}}$ is maximal if and only if there is a prime $p$ such that for all $G,\left|G / G_{\underline{\underline{X}}}\right|$ is 1 or $p$. From [3] a maximal Fitting class is normal, and hence $\underline{\underline{X}}$ is a maximal Fitting class if and only if there exists a prime $p$ such that $\left|G / G_{\underline{\underline{x}}}\right|$ is 1 or $p$ for all $G$. This does not hold in general, but we can prove a similar result.

THEOREM 1.1. Let $\underline{\underline{X}}, \underline{\underline{Y}}$ be Fitting classes with $\underline{\underline{X}} \subset \underline{\underline{Y}}$. Then

(i) if for all $G \in \underline{\underline{Y}}$, and some prime $p$, the $\underline{\underline{X}}$-injectors of $G$ have index 1 or $p$, then $\underline{\underline{X}}$ is normal and maximal in $\underline{\underline{Y}}$, and

(ii) if $\mathrm{X}$ is maximal in $\underline{\underline{\mathrm{Y}}}$, then there is a prime $p$ such that $G / G_{\underline{\underline{X}}}$ is a p-group for all $G \in \underline{\underline{Y}}$.

It seems difficult to close the gap between $(i)$ and $(i i)$. Let $p$ and $q$ be distinct primes, $\stackrel{\underline{S}}{=}\left(\underline{\underline{S}}_{q}\right)$ the class of $p$-groups (q-groups), $\underline{N}\{p, q\}$ the class of nilpotent $\{p, q\}$-groups, and $\underline{S} \underline{\underline{S}} \underline{S} q$ the usual product of the classes $\underline{\mathrm{S}}_{p}$ and $\underline{\underline{S}}_{q}$. Then if $\underline{\underline{\mathrm{x}}}=\underline{\underline{\mathrm{S}}} p, \underline{\underline{\mathrm{Y}}}=\underline{\underline{\mathrm{N}}}\{p, q\}, \underline{\underline{\mathrm{X}}}$ is maximal in $\underline{\underline{Y}}$, but we can clearly obtain any $q$-group as $G / G \underline{\underline{x}}$ for some $G \in \underline{Y}$. Hence $(i)$ is not necessary. On the other hand (ii) is not sufficient, for if $\underline{\underline{\mathrm{X}}}=\underline{\underline{\mathrm{S}}}_{p}, \underline{\underline{\mathrm{Y}}}=\underline{\underline{\mathrm{S}}} \underline{\underline{\underline{\mathrm{S}}}} q$, we have $G / G_{\underline{\underline{\mathrm{X}}}} \in \underline{\underline{\mathrm{S}}}_{q}$ for any $G \in \underline{\underline{Y}}$, but $\underline{\underline{X}} \subset \underline{\underline{N}}\{p, q\} \subset \underline{\underline{Y}}$.

We give a negative answer to the second question by showing that there 
are normal Fitting classes not contained in any maximal Fitting class. We do this by exploiting the normal Fitting pairs of Lausch [6].

Let $A$ be an abelian group (not necessarily finite), and hom( $\underline{\underline{S}}, A$ ) the family of all group homomorphisms from groups in $\underline{\underline{\mathrm{S}}}$ to $A$. Let $d: \underline{\underline{\mathrm{S}}} \rightarrow \operatorname{hom}(\underline{\underline{S}}, A)$ be a function satisfying

$$
G d: G \rightarrow A,
$$

and, whenever $\alpha: G_{1} \rightarrow G_{2}$ is a subnormal embedding,

$$
G_{1} d=\alpha\left(G_{2} d\right)
$$

Under these conditions, Lausch proves that the set $\{g(G d): G \in \underline{\underline{\mathrm{S}}}, g \in G\}$ is a subgroup of $A$. If this subgroup is $A$ itself, we call the pair $(A, d)$ a normal Fitting pair. Recalling that Blessenohl and Gaschütz [1] establish the existence of a urique minimal normal Fitting class, $\underline{\underline{H}}$ say, the following statement summarises the results of Lausch we need.

1.2. There exists a normal Fitting pair $(A, d)$ such that

$$
\underline{\underline{H}}=\{G: G(G d)=I\} \text {. }
$$

Further, if $\underline{\underline{X}}$ is a normal Fitting class, then

$$
X=\{g(G d): g \in G, G \in \underline{\underline{X}}\}
$$

is a subgroup of $A$, and if $Y$ is a subgroup of $A$, then

$$
\underline{\underline{\mathrm{Y}}}=\{G: G(G d) \leq Y\}
$$

is a normal Fitting class. If we consider normal Fitting classes and the subgroups of $A$ both ordered by inclusion, then the map $\underline{\underline{X}} \rightarrow X$ is an order isomorphism. If $v: A \rightarrow A / X$ is the natural epimorphism, then $(A / X, d v)$ is a normal Fitting pair.

It follows that $\underline{\underline{X}}$ will be contained in no maximal normal Fitting class if $A / X$ contains no maximal subgroups. Thus it will be sufficient to show that $A$ has a p-quasicyclic quotient group for some prime $p$. Rather than prove this directly, we observe that it follows immediately from the following theorem, which sheds more light on the structure of Lausch's group $A$ in 1.2. Let $P$ be the set of prime numbers, $N$ the set of positive integers, and $C_{n}$ the cyclic group of order $n$.

THEOREM 1.3. Let $X$ denote the (restricted) direct product 
$\prod\left\{C_{p}{ }_{p}: p \in P, \alpha \in N\right\}$. Then $A$ has a direct factor isomorphic to the (restricted) direct product of countably many copies of $X$.

\section{Proof of Theorem 1.1}

(i) Suppose first that $\underline{\underline{X}}$ is not normal in $\underline{\underline{Y}}$. Then there is a group $G \in \underline{\underline{Y}} \underline{\underline{X}}$ such that the $\underline{\underline{X}}$-injectors of $G$ are not normal in $G$. Let $I$ be an $\underline{x}$-injector of $G:$ then $|G: I|=p$. Since $I$ is a maximal subgroup of $G, G /$ core $I$ is primitive, and has a unique minimal normal subgroup of order $p$. Thus $G /$ core $I=D_{p}^{r}$, and $G_{\underline{\underline{X}}}=$ core $I$ (where $D_{p}^{r}$ is the group with a unique minimal normal subgroup $N$ of exponent $p$,

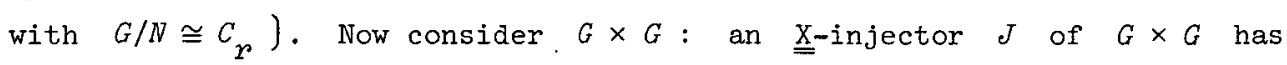
index $P$, and contains $G_{\underline{\underline{X}}} \times G_{\underline{\underline{X}}}^{\cdot} \cdot$ Thus $\delta /\left(G_{\underline{X}} \times G_{\underline{X}}\right)$ is isomorphic to a subgroup of index $p$ in $D_{p}^{r} \times D_{p}^{r}$. An easy calculation shows that such a subgroup must contain one component subgroup. But this implies that in $G \times G$, one of the component subgroups is contained in $J$, giving $G \in \underline{\underline{X}}$, a contradiction. Hence $\underline{\underline{X}}$ is normal in $\underline{\underline{Y}}$.

Now suppose that $\underline{\underline{X}}$ is not maximal in $\underline{\underline{Y}}$. Then there is a Fitting

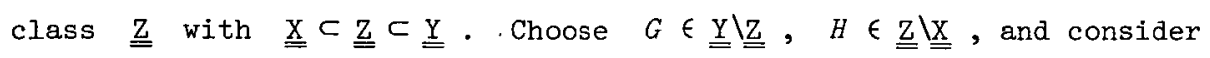
$(G \times H)_{\underline{\underline{\mathrm{X}}}}$. Since $G \times H \in \underline{\underline{\underline{\mathrm{Y}}}} \underline{\underline{\mathrm{X}}},(G \times H)_{\underline{\underline{\mathrm{X}}}}$ has index $p$ in $G \times H$. Since neither $G$ nor $H$ is in $\underline{\underline{X}}$, the only possibility is that $(G \times H)_{\underline{\underline{X}}} /\left(G_{\underline{\underline{X}}} \times H_{\underline{X}}\right)$ is a diagonal subgroup of $\left(G / G_{\underline{\underline{X}}}\right) \times\left(H / H_{\underline{\underline{X}}}\right)$. But then $(G \times H)_{\underline{\underline{X}}} / G_{\underline{X}} \cong H \in \underline{\underline{Z}}$, $G_{\underline{\underline{X}}} \cap H_{\underline{\underline{X}}}=1,(G \times H)_{\underline{\underline{X}}} /\left(G_{\underline{\underline{X}}} \times H_{\underline{\underline{X}}}\right)$ is nilpotent and so from Lemma 1.2 of [2], $G \cong(G \times H) \underline{\underline{X}}^{\prime} \underline{\underline{X}} \in \underline{\underline{Z}}$, a contradiction. Hence $\underline{\underline{X}}$ is maximal in $\underline{\underline{Y}}$.

(ii) Suppose that $\underline{\underline{X}}$ is maximal in $\underline{\underline{Y}}$, and let $G$ be of minimal order with $G \in \underline{\underline{Y}} \underline{\underline{X}}$. Then $\left|G / G_{\underline{\underline{X}}}\right|=p$ for some prime $p$. Suppose there is a group $H \in \underline{\underline{\mathrm{Y}} \underline{\underline{\mathrm{X}}}}$ such that $H / H_{\underline{\mathrm{X}}}$ is not a p-group. Let $\underline{\underline{\mathrm{XS}}}$ be the product of $\underline{\underline{X}}$ and $\underline{S_{p}}$ : it is easy to check that $\underline{\underline{X S}}_{p}$ is a Fitting class, and $\underline{\underline{X} \subseteq \underline{\underline{X S}}} p$. Then we have $\underline{\underline{X}} \subset \underline{\underline{X S}} p \cap \underline{\underline{Y}}$, since $G \in(\underline{\underline{X S}} p \underline{\underline{Y}}) \underline{\underline{X}}$, and $\underline{\underline{X S}} \cap \underline{\underline{Y}} \subset \underline{\underline{Y}}$, since $H \in \underline{\underline{\underline{Y}}} \backslash \underline{\underline{X S}} \underline{\underline{X}} \cap \underline{\underline{Y}})$. Thus $\underline{\underline{X}}$ is not maximal in $\underline{\underline{Y}}$, 
a contradiction.

The normality follows immediately from the fact that if $G \in \underline{\underline{Y}}, G$

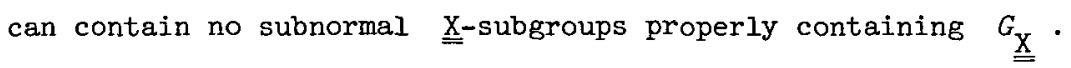

\section{The proof of Theorem 1.3}

We begin with the following result.

LEMMA 3.1. For each prime $p$ and each positive integer $\alpha$, the set

$$
I(p, \alpha)=\left\{q \in P: p^{\alpha} \| q-1\right\}
$$

is infinite, where $p^{\alpha} \mid q-1$ means $p^{\alpha} \mid q-1, p^{\alpha+1} \backslash q-1$.

Proof. By a result of Dirichlet (Theorem 15 in [5]) there are infinitely many integers $n$ for which $p^{\alpha+1} n+p^{\alpha}+1$ is prime, and hence infinitely many $n$ for which $p^{\alpha+1} n+p^{\dot{\alpha}}+1 \in I(p, \alpha)$.

We now choose normal Fitting classes as follows. Let $p \in P$, $\alpha \in N, q \in I(p, \alpha)$, and let $A(p, \alpha, q)$ be the Sylow $p$-subgroup of the multiplicative group $A(q)$ of the field of $q$ elements, so that $A(p, \alpha, q)$ is cyclic of order $p^{\alpha}$; let $a(p, \alpha, q)$ be a generator of $A(p, \alpha, q)$. Blessenohl and Gaschütz [1] have shown that if, for each $G \in \underline{\underline{\mathrm{S}}}$, the function $G \delta: G \rightarrow A(q)$ is defined by

$$
g(G \delta)=\prod_{H / K} \operatorname{det}_{H / K} g
$$

(the product of the determinants of the linear transformations induced by $g$ on the $q$-chief factors $H / K$ in some chief series of $G$ ), then $(A(q), \delta)$ is a normal Fitting pair. Hence if $d(p, \alpha, q): \underline{\underline{\mathrm{s}}} \rightarrow \operatorname{hom} \underline{\underline{\mathrm{s}}}, A(p, \alpha, q))$ is defined by $G d(p, \alpha, q)=(G \delta) \nu$, where $\nu$ is the natural epimorphism $A(q) \rightarrow A(p, \alpha, q)$,

$$
(A(p, \alpha, q), d(p, \alpha, q)) \text { is a normal Fitting pair. }
$$

Note that if $G=D_{q}^{p^{\alpha}}, q \in I(p, \alpha)$, then $G(G d(p, \alpha, q))=A(p, \alpha, q)$.

The next result is easy to prove: we omit the proof.

THEOREM 3.3. Let $\left(A_{i}, d_{i}\right) \quad(i=1,2, \ldots)$ be a collection of 
normal Fitting pairs with the property that for all $G \in \underline{\underline{S}}$,

$$
\left|\left\{i: G\left(G d_{i}\right) \neq I\right\}\right|<\infty \text {. }
$$

For each $G \in \underline{\underline{S}}$, define a function $G d_{0}: G \rightarrow \prod A_{i}$ by, for $g \in G$,

$$
g\left(G d_{0}\right)(i)=g\left(G d_{i}\right)
$$

Then $A_{0}=\left\{g\left(G d_{0}\right): g \in G, G \in \underline{\underline{s}}\right\}$ is a subgroup of $\prod A_{i}$, and $\left(A_{0}, d_{0}\right)$ is a normal Eitting pair. Indeed, $A_{0}$ is subdirect in $\prod A_{i}$.

Since only finitely many of the maps $G d(p, \alpha, q)$ can give $G(G d(p, \alpha, q)) \neq I$ for a given $G$,

$$
\{(A(p, \alpha, q), d(p, \alpha, q)): p \in P, \alpha \in N, q \in I(p, \alpha)\}
$$

satisfies the condition of Theorem 3.3. Put

$$
A^{*}=\prod\{A(p, \alpha, q): p \in P, \alpha \in \alpha, q \in I(p, \alpha)\}
$$

and define $d^{*}: \underline{\underline{\mathrm{S}}} \rightarrow \operatorname{hom}\left(\underline{\underline{S}}, A^{*}\right)$ as in Theorem 3.3. We prove

3.4. $\left(A^{*}, d^{*}\right)$ is a normal Fitting pair.

We need only show $A^{*}=A_{0}=\left\{g\left(G d^{*}\right): g \in G, G \in \underline{\underline{S}}\right\}$. To do this we show that $A_{0}$ contains a generating set for $A^{*}$. For $p \in P, \alpha \in N$, $q \in I(p, \alpha)$ put $G=D_{q}^{p^{\alpha}}$, and compute $G\left(G d^{*}\right)$. Clearly, if $q^{\prime} \neq q$,

$$
G\left(G d\left(p^{\prime}, \alpha^{\prime}, q^{\prime}\right)\right)=1 ;
$$

and so suppose $q=q^{\prime}$ and consider $G\left(G d\left(p^{\prime}, \alpha^{\prime}, q\right)\right)$. If $p \neq p^{\prime}$, then the definition of $A\left(p^{\prime}, \alpha^{\prime}, q\right)$ gives $G\left(G d\left(p^{\prime}, \alpha^{\prime}, q\right)\right)=1$. If $p=p^{\prime}, \alpha \neq \alpha^{\prime}$, then by their definition $I(p, \alpha) \cap I\left(p, \alpha^{\prime}\right)=\emptyset$, and so $\alpha=\alpha^{\prime}$. Finally, $G(G d(p, \alpha, q))=A(p, \alpha, q)$, and hence

$$
G\left(G d^{*}\right)=A(p, \alpha, q) \text {. }
$$

Thus $A_{0}$ contains $A(p, \alpha, q)$ for all relevant $(p, \alpha, q)$ : since $A^{*}$ is generated by these, we have $A_{0}=A^{*}$.

Now $A^{*}$ is a homomorphic image of Lausch's group $A$, say $\mu: A \rightarrow A^{*}:$ then $d \mu=d^{*}$. 
If $G=D_{q}^{p^{\alpha}}, p \in P, \alpha \in N, q \in I(p, \alpha)$, then $G^{\prime}(G d)=1$, and so $G(G d)$ is cyclic of order $p^{\alpha}$, generated by $b(p, \alpha, q)$ say. Then the map $v$ defined by

$$
\nu: a(p, \alpha, q) \rightarrow b(p, \alpha, q)
$$

is a monomorphism, and $\nu \mu$ is the identity map on $A^{*}$. It follows in the usual manner that

$$
A \cong \operatorname{ker} \mu \times A^{*}
$$

But $A^{*}$ is isomorphic to the restricted direct product of a countable number of copies of $\prod\left\{C_{p^{\alpha}}: p \in P, \alpha \in N\right\}$, and Theorem 1.3 is proved.

\section{References}

[1] Dieter Blessenohl und Wolfgang Gaschütz, "Über normale Schunk- und Fittingklassen", Math. Z. 118 (1970), 1-8.

[2] R.A. Bryce and John Cossey, "Metanilpotent Fitting classes", J. Austraz. Math. Soc. (to appear).

[3] John Cossey, "Products of Fitting classes", in preparation.

[4] B. Fischer, W. Gaschütz, und B. Hartley, "Injektoren endlicher auflösbarer Gruppen", Math. Z. 102 (1967), 337-339.

[5] G.H. Hardy and E.M. Wright, An introduction to the theory of numbers, 4th ed. (Clarendon Press, Oxford, 1960; reprinted 1971).

[6] Hans Lausch, "On normal Fitting classes", Math. Z. 130 (1973), 67-72.

Department of Pure Mathematics,

School of General Studies,

Australian National University,

Canberra, ACT. 